Dieses Dokument ist eine Zweitveröffentlichung (Postprint) /

This is a self-archiving document (accepted version):

Felix Winkler, Milan Pešić, Claudia Richter, Michael Hoffmann, Thomas Mikolajick, Johann W. Bartha

Demonstration and Endurance Improvement of p-channel Hafniabased Ferroelectric Field Effect Transistors

Erstveröffentlichung in / First published in:

Device Research Conference (DRC). Ann Arbor, 2019. IEEE, S. 51-52. ISBN 978-1-7281-2112-3

DOI: https://doi.org/10.1109/DRC46940.2019.9046463

Diese Version ist verfügbar / This version is available on:

https://nbn-resolving.org/urn:nbn:de:bsz:14-qucosa2-775643 


\title{
Demonstration and Endurance Improvement of p-channel Hafnia-based Ferroelectric Field Effect Transistors
}

\author{
Felix Winkler ${ }^{1, *}$, Milan Pešić2,\#,*, Claudia Richter ${ }^{3}$, Michael Hoffmann ${ }^{3}$, Thomas Mikolajick ${ }^{1,3}$, Johann W. Bartha ${ }^{1}$ \\ ${ }^{1}$ Technische Universität Dresden, Institute of Semiconductors and Microsystems, IHM, Dresden, Germany \\ ${ }^{2}$ MDLSoft Inc., Santa Clara, CA, USA; "Now with Applied Materials, Santa Clara, CA, USA \\ ${ }^{3}$ Namlab gGmbH, Noethnitzer Str. 64, 01187 Dresden, Germany \\ Felix.Winkler1@tu-dresden.de,milan_pesic@amat.com,*both authors equally contributed
}

Introduction: So far, only CMOS compatible and scalable hafnia-zirconia (HZO) based ferroelectric (FE) n-FeFETs have been reported. To enable the full ferroelectric hierarchy [1] both p- and n-type devices should be available. Here we report a p-FeFET with a large memory window (MW) for the first time. Moreover, we propose different integration schemes comprising structures with and without internal gate resulting in metal-FE-insulator-Si (MFIS) and metalFE-metal-insulator-Si (MFMIS) devices which could be used to tackle the problem of interface (IF) degradation and possibly decrease the power consumption of the devices.

Device Fabrication: The p-channel FeFETs with and without internal gate with gate lengths ranging from 10 to 25 $\mu \mathrm{m}$ were fabricated based on the integration scheme showed in Fig. 1a-d. A photograph of the transistors can be seen in Fig. 1e and the process sequence for the test devices is shown in Fig. 1f.

Results and Discussion: As shown in Fig. 2a-d, the FE properties of the devices were investigated using first order reversal curves (FORC) [2] which pointed to a slight biasing of the structure (due to the asymmetry of the TiN work function and Si substrate) with a very tight distribution of the bias field. The devices exhibited very clean, leakagefree polarization-voltage $(\mathrm{P}-\mathrm{V})$ curves showing the same bias as expected form the FORC measurements. Well saturated $P-V$ characteristics with a $P_{r}$ of $17.5 \mu \mathrm{C} / \mathrm{cm}^{2}$ and wake-up-free [3] field cycling was observed. Beside the MFMIS transistors, processed MFMIS capacitor test-structures exhibited similar $P_{r}$ values and well saturated characteristics.

Large test structures resulted in very good transistor behavior (Fig. 3a) with very low gate leakage (Fig. 3a/d). Table 1 shows different process conditions. As expected, an increase of the IF thickness resulted in leakage reduction. $I_{D}-V_{G}$ curves and the hysteresis of a $C-V$ sweep unveiled a MW $>2 \mathrm{~V}$ (a record of $>80 \%$ of theoretical MW) (Fig $3 \mathrm{~b} / \mathrm{c}$ ).

The IF and its thickness play a crucial role in the functioning of FeFETs as most of the applied voltage (during program and erase operation; PRG and ERS, respectively) drops over it [4-6]. This increases PRG / ERS voltages, degrades the interface [4-6] and causes a depolarization field, which destabilizes the stored memory state [5]. Only devices with an IF thickness below $4 \mathrm{~nm}$ showed a FE MW, others resulted in a negative MW due to trapping and depolarization (Fig. 4a) [5]. Fig. 4c and 4d show a larger MW and higher retention for the thinner IF (native oxide) and a stronger relaxation of $P_{r}$ for the thicker IF (chemically grown). The majority of the devices with internal gate showed a problem with internal gate isolation (high leakage) and could not be evaluated. A simulations study was carried out instead, using the commercially available multi-scale simulation tool Ginestra ${ }^{T M}$ [7], already proven in assessing FE devices $[4,8]$.

Gate Stack Engineering: As aforementioned, the IF and FE-HZO are forming a voltage divider. Area factor (area of internal vs. area of control gate) tuning [9] using an internal gate can be employed to modify the voltage distribution across the voltage divider (same effect as changing the k-value of the $\mathrm{SiO}_{2} \mathrm{IF}$ ), thus reducing the field loss and stress over the critical interfacial layer. Its impact on the MW - when polarization is fully saturated - is negligible (Fig. 5a) for the case of the $1.6 \mathrm{~nm}$ thick IF below the floating gate. However, to saturate $P_{r}$, voltages above $8 \mathrm{~V}$ are required, which would result in instantaneous breakdown of the IF of the device. Therefore, a realistic operation voltage of 5.6 $\mathrm{V}$ was used for modeling and we estimated the possible decrease of the operation conditions and field over the stack (Fig. 5b) depending on the area factor while preserving the MW. An area ratio of 5, which could be realized in a recessed gate integration scheme, reduces the programming voltage by more than $40 \%$ to $3.2 \mathrm{~V}$ and below (Fig. $5 \mathrm{~b}$ ). This approach is very promising for reducing the degradation of the IF and achieving low power devices with enhanced endurance.

Conclusion: In this study, we show the first p-FeFET with an excellent MW of more than $2 \mathrm{~V}$, low leakage and very high $I_{O N} / I_{O F F}$ ratio. An integration scheme with floating gate and tuning of the area factor of the FeFET was proposed. The proposed gate stack engineering of the voltage divider enables lower programming voltage and a reduction of the field over the interface by a factor of 3 . An increase of cycling endurance is therefore expected.

References: [1] Mueller et al IMW 2018. [2] Schenk et al. ACS Adv.Mat.\&Int. 2016. [3] Pesic et al. Adv.Func.Mat. 2016. [4] Pesic et al. IEEE-IEDM 2018. [5] Pesic et al. IEEE-TDMR 2018. [6] Yurchuk et al. IEEE-TED 2016. [7] MDLSoft Inc. www.mdlsoft.com. [8] Pesic IEEE-JEDS 2018. [9] Mueller et al. NVMTS 2016. [10] Strobel et al. Sol.Eng. Mat \&Sol. cell 2015. 


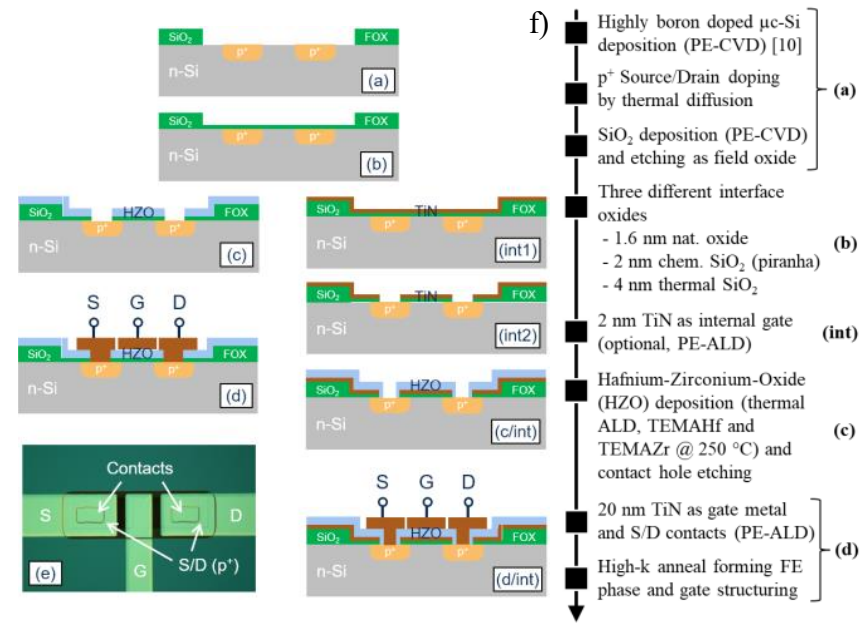

Fig. 1. a-d) Integration flow of the devices with and without internal gate. e) Design of the transistors (top view microscope photograph). f) Process sequence

Table. 1. Process conditions A - F with interface thickness $d_{I F}$, HZO thickness $d_{H Z O}$ and annealing conditions Anneal.
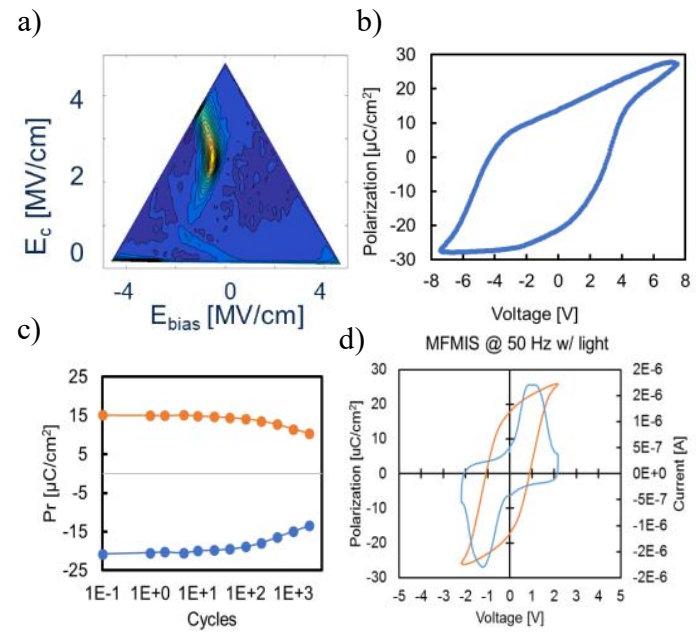

Fig. 2. a) FORC Preisach density. b) Saturated $P$ - $V$ curve and c) $P_{r}$-endurance of the p-FeFET. d) $P-V$ characteristics of MFMIS.

\begin{tabular}{|l|c|c|c|c|c|c|}
\hline Process: & A & B & C & D & E & F \\
\hline$d_{I F}$ & $1.6 \mathrm{~nm}$ & $2 \mathrm{~nm}$ & $4 \mathrm{~nm}$ & $4 \mathrm{~nm}$ & $4 \mathrm{~nm}$ & $4 \mathrm{~nm}$ \\
\hline$d_{\text {HZO }}$ & $15 \mathrm{~nm}$ & $15 \mathrm{~nm}$ & $16 \mathrm{~nm}$ & $16 \mathrm{~nm}$ & $24 \mathrm{~nm}$ & $24 \mathrm{~nm}$ \\
\hline \multirow{2}{*}{ Anneal } & $600{ }^{\circ} \mathrm{C}$ & $600^{\circ} \mathrm{C}$ & $650^{\circ} \mathrm{C}$ & $1000^{\circ} \mathrm{C}$ & $650^{\circ} \mathrm{C}$ & $1000^{\circ} \mathrm{C}$ \\
& $20 \mathrm{~s}$ & $20 \mathrm{~s}$ & $20 \mathrm{~s}$ & $1 \mathrm{~s}$ & $20 \mathrm{~s}$ & $1 \mathrm{~s}$ \\
\hline
\end{tabular}

a)

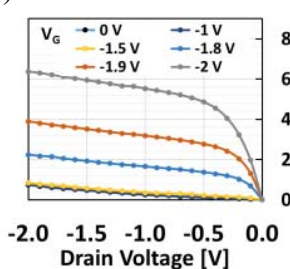

b)

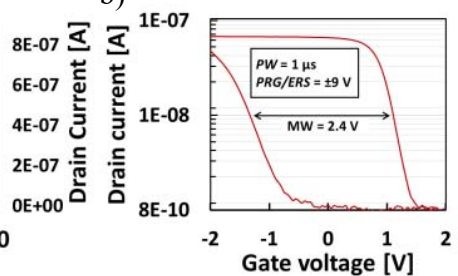

c)

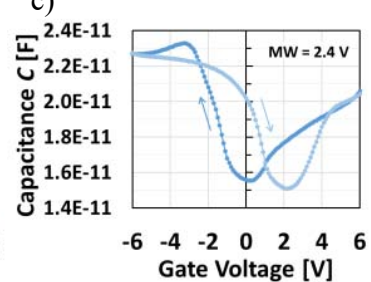

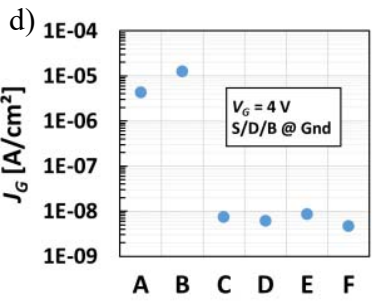

Fig. 3. a) $I_{D^{-}} V_{D}$ characteristics of the static readout of the cell after PRG. b) $I_{D^{-}} V_{G}$ shift due to programing and erasing and the corresponding $\mathrm{MW}=2.4 \mathrm{~V}$ c) $C$ - $V$ sweep d) $J_{G}$ measured at $V_{G}=4 \mathrm{~V}$ for processes $\mathrm{A}-\mathrm{F}$.
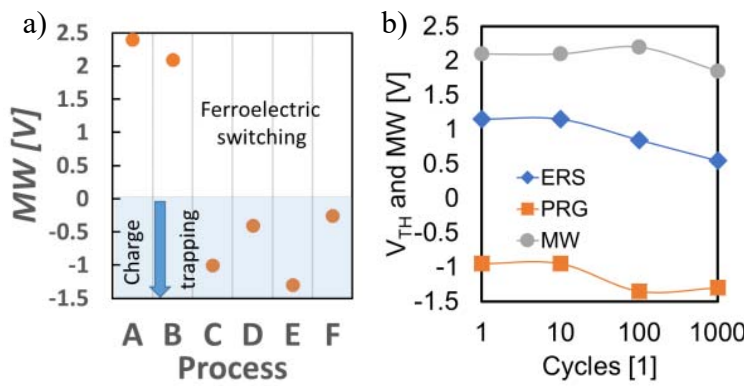

c) retention @ $85^{\circ} \mathrm{C}$

d)
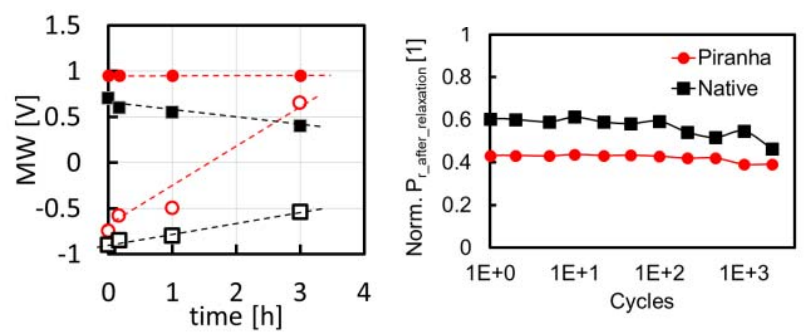

Fig. 4. Memory properties of the p-FeFETs. a) MW as function of the process condition. Comparison of the b) endurance, c) retention and d) $P_{r}$-evolution with cycling for transistor test structures with native oxide (black) and chemically grown (red) interfacial buffer oxide.

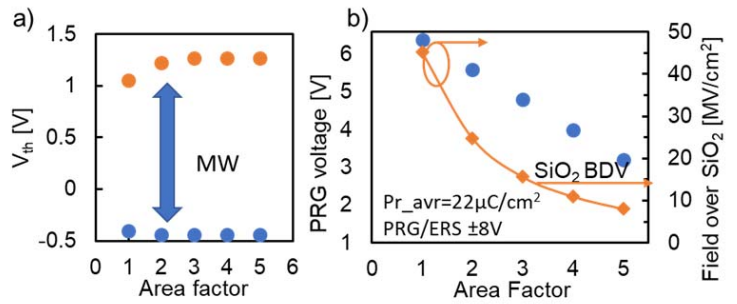

Fig. 5. a) Simulated MW of the FeFET depending on the area factor (saturated case). An interface layer thickness below the floating electrode of $1.6 \mathrm{~nm}$ was assumed. b) PRG voltage and field over interface reduction as a function of the area factor. 